\title{
Single spontaneous photon as a coherent beamsplitter for an atomic matter-wave
}

\author{
Jiří Tomkovič ${ }^{1 \star}$, Michael Schreiber ${ }^{2}$, Joachim Welte ${ }^{1}$, Martin Kiffner $^{3}$, Jörg Schmiedmayer ${ }^{4}$ \\ and Markus K. Oberthaler ${ }^{1}$
}

In spontaneous emission an atom in an excited state undergoes a transition to the ground state and emits a single photon. Associated with the emission is a change of the atomic momentum due to photon recoil'. Photon emission can be modified close to surfaces ${ }^{2,3}$ and in cavities ${ }^{4}$. For an ion, localized in front of a mirror, coherence of the emitted resonance fluorescence has been reported ${ }^{5,6}$. Previous experiments demonstrated that spontaneous emission destroys motional coherence ${ }^{7-9}$. Here we report on motional coherence created by a single spontaneous emission event close to a mirror surface. The coherence in the free atomic motion is verified by atom interferometry ${ }^{\mathbf{1 0}}$. The photon can be regarded as a beamsplitter for an atomic matter-wave and consequently our experiment extends the original recoiling slit Gedanken experiment by Einstein ${ }^{11,12}$ to the case where the slit is in a robust coherent superposition of the two recoils associated with the two paths of the quanta.

We consider an atom passing by a mirror which spontaneously emits a single photon (see Fig. 1a). As a result of the photon momentum the atom receives a corresponding recoil kick in the direction opposite to the photon emission. In the absence of the mirror the observation of the emitted photon direction implies the knowledge of the atomic momentum resulting from the photonatom entanglement ${ }^{8}$. In the presence of the mirror the detection of a photon in a certain direction does not necessarily reveal if it has reached the observer directly or via the mirror. For the special case of spontaneous emission perpendicular to the mirror surface the two emission paths are in principle indistinguishable for small atom-mirror distances $d \ll c / \Gamma$, where $c$ is the speed of light and $\Gamma$ the natural linewidth. This general limit is always fulfilled in our experiments. Thus the atom after this emission event is in a superposition of two motional states.

This is also true for the more general case of tilted emission, as revealed in Fig. $1 \mathrm{~b}$ for emission close to the mirror surface. One expects residual coherence for emission angles where the optical absorption cross-section of the atom and the mirror atom observed by a fictitious observer in the emission direction still overlap. This is visualized in Fig. 1b, where the corresponding cross-sections are indicated with the bars. The overlap as a function of emission direction is depicted on the sphere (blue no coherence, red full coherence). The result on the atomic motion is indicated for one special trajectory which starts with an atom moving parallel to the mirror surface and a single photon emission at an angle to the mirror normal. This case leads to an imperfect coherent superposition of two momentum states separated by less than two photon momenta $\hbar k_{0}$. The spatial distribution of the atoms at the position of the detector is shown, where the colour corresponds to the degree of coherence. In Fig. 1c we contrast this to the case of a larger distance to the mirror, where the portion of coherent atomic momentum is strongly reduced.

It is important to keep in mind that a single particle detector cannot distinguish between coherent superpositions and mixtures but only gives the probability distribution. Thus an interferometric measurement ${ }^{13}$ has to be applied to reveal the expected coherent structure (see Fig. 2). For that, the two momentum states of interest have to be overlapped and the coherence (that is, well-defined phase difference) is verified by observing an interference pattern as a function of a controlled phase shift applied to one of the momentum states. The two outermost momentum states are expected to show the highest coherence. Their recombination can be achieved by a subsequent Bragg scattering off an independent standing light wave (see Fig. 2b) with a suitable wavelength ${ }^{10,14}$. The relative phase $\phi_{\mathrm{B}}$ is straightforwardly changed by shifting the probing standing light wave. This is implemented by moving the retroreflecting mirror by distance $L$. The upper graph depicts the results obtained for large distances $(>54 \mu \mathrm{m})$ of the atom to the mirror (that is, a free atom). In this case no interference is observed, and thus spontaneous emission induces a fully incoherent modification of the atomic motion. For a mean distance of $2.8 \mu \mathrm{m}$, clear interference fringes are observed, demonstrating that a single spontaneous emission event close to a mirror leads to a coherent superposition of outgoing momentum states.

In the following we describe the essential parts of the experimental set-up shown in Fig. 2b, lower graph. Further details are provided in the Supplementary Information. As the effect depends critically on the distance between the atom and mirror, a well-collimated and localized beam of ${ }^{40} \mathrm{Ar}$ atoms in the metastable $1 s_{5}$ state is used. To ensure the emission of only a single photon we induce a transition $1 s_{5} \rightarrow 2 p_{4}\left(\lambda_{\mathrm{E}}=715 \mathrm{~nm}\right)$. From the excited state $2 p_{4}$, the atom predominantly decays to the metastable $1 s_{3}$ state via spontaneous emission of a single photon $\left(\lambda_{\mathrm{SE}}=795 \mathrm{~nm}\right)$ (branching ratio of $\left.1 s_{5} / 1 s_{3}=1 / 30\right)$. The residual $1 s_{5}$ are quenched to an undetectable ground state by an additional laser. Choosing the appropriate polarization of the excitation laser the atomic dipole moment is aligned in the mirror plane, leading to the momentum distribution after spontaneous emission shown in Fig. 2a. The interferometer is realized with a far-detuned standing light wave on a second mirror. Finally the momentum distribution is detected by a spatially resolved multi channel plate $(\mathrm{MCP}) \approx 1 \mathrm{~m}$ behind the spontaneous emission, enabling different momenta to be distinguished.

\footnotetext{
${ }^{1}$ Kirchhoff-Institut für Physik, Universität Heidelberg, Im Neuenheimer Feld 227, 69120 Heidelberg, Germany, ${ }^{2}$ Ludwig-Maximilians-Universität, Schellingstr. 4, 80799 München, Germany, ${ }^{3}$ Physik Department I, Technische Universität München, James-Franck-Straße, 85747 Garching, Germany,

${ }^{4}$ Vienna Center for Quantum Science and Technology, Atominstitut, TU Wien, 1020 Vienna, Austria. *e-mail: single-photon@matterwave.de.
} 


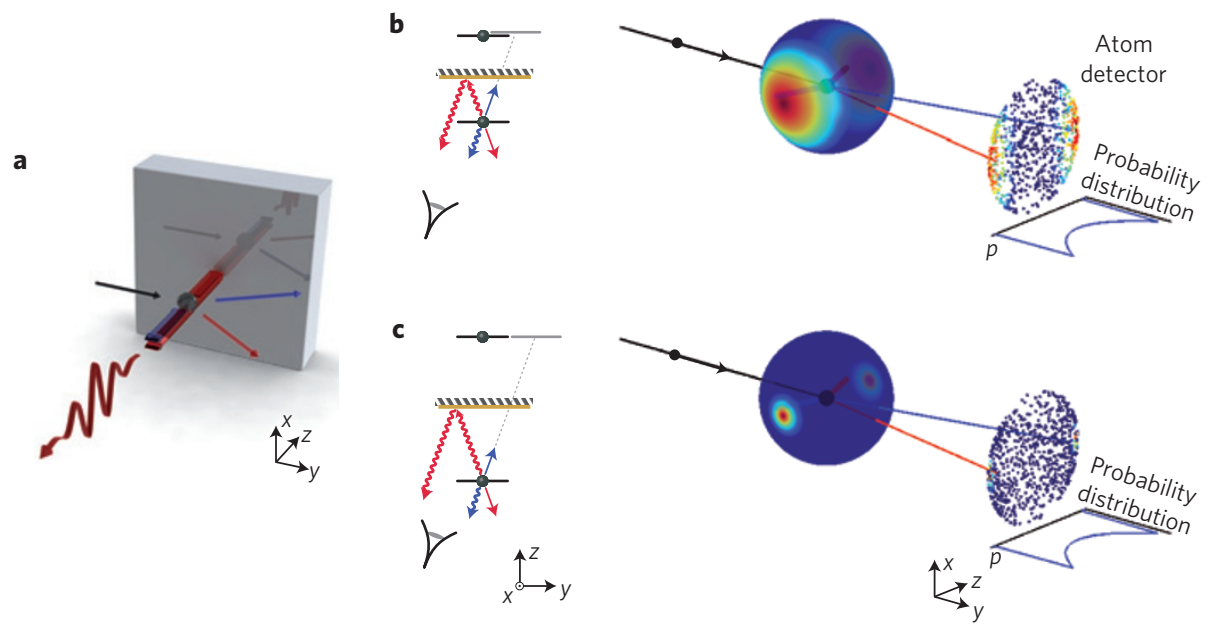

Figure 1 | Motional coherence generated by a single spontaneous emission event. a, The situation of interest is depicted-an atom in front of a mirror spontaneously emits a single photon. For emission perpendicular to the mirror surface an observer can in principle not distinguish if the photon has been reflected or not. Momentum conservation in the atom-photon system implies that the atom after the emission is in a coherent superposition of two different momentum states separated by twice the photon recoil. $\mathbf{b}$, Indistinguishability is also given for more general emission directions. With the spatial extension of the atom corresponding to the optical absorption cross-section, indistinguishability can be estimated by the projected overlap of the atom and its mirror image. This overlap is represented by a colour code on a sphere for all emission directions (red: full coherence, blue: no coherence). Repeating the experiment-a single atom emits a single photon-leads to the indicated pattern at the atom detector. The colour code indicates the probability of generating a coherent superposition for the corresponding event (red: full coherence, blue: no coherence). c, In the case of large distances to the mirror, the coherent portion is drastically reduced, approaching the limit of vanishing coherence in free space.

a
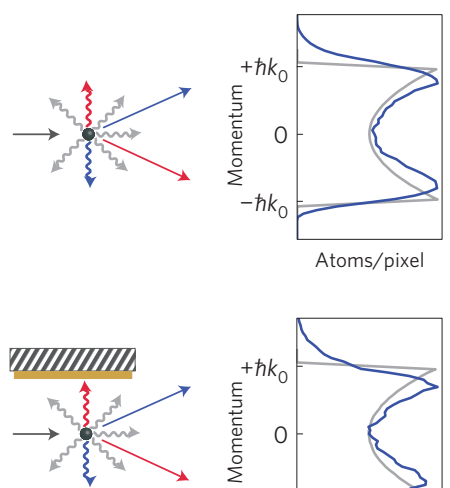
$\uparrow^{z} \rightarrow y$

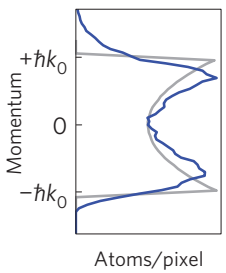

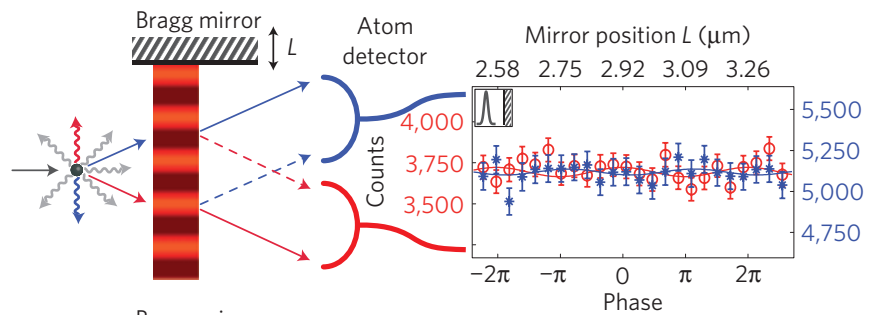

Mirror position $L(\mu \mathrm{m})$

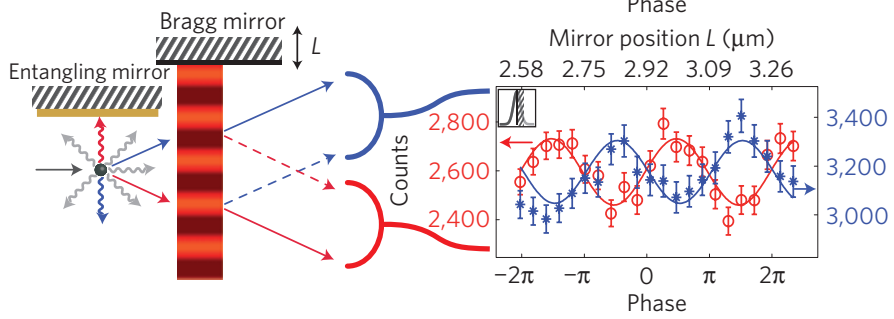

Figure 2 | Experimental confirmation of coherence induced by spontaneous emission. a, Experimental observation of the momentum distribution does not reveal the coherence. In both cases-close to and far from the mirror-the momentum distribution is the same (blue line). To compare the observed momentum distribution after spontaneous emission with theory (light grey) the data has been deconvoluted by the initial momentum distribution. The deviation results from a residual filtering of high spatial frequencies. $\mathbf{b}$, The coherence is revealed if the spontaneous emission event is employed as the first beamsplitter of an atom interferometer. The recombination is accomplished by Bragg scattering from a standing light wave. The relative phase of the two paths can be changed by moving the 'Bragg' mirror as indicated. In the case of a mean distance of $54 \mu \mathrm{m}$ between atoms and 'entangling' mirror (upper graph, error bars indicate Poisson noise) no interference signal is observed, confirming the free space limit. The inset depicts the position of the 'entangling' mirror with respect to the atomic beam. For a mean distance of $2.8 \mu \mathrm{m}$ (lower graph) the two complementary outputs of the interferometer reveal an interference pattern with a maximal visibility of $5.9 \% \pm 1.1 \%$.

For systematic studies of the coherence we analyse the probability of finding a particle in a coherent superposition of momentum states as a function of atom-mirror distance $d$. This is done by analysing the final momentum distribution for different phases $\phi_{\mathrm{B}}$ within the interferometer and fitting to each resolved momentum $(\approx 1 / 8$ of a photon momentum) an interference pattern given by

$$
N=N_{0}+N_{A} \cos \left(\phi_{\mathrm{B}}+\phi_{0}\right)
$$

In Fig. 3 we plot the visibility $V=N_{\mathrm{A}} / N_{0}$ (with $N_{0}$ the constant atom number and $N_{\mathrm{A}}$ the oscillatory part) revealing that the coherence vanishes within distances of a few micrometres from the mirror.

For a basic understanding of the physics behind the experimental observation we use a simple semiclassical model. We follow the picture of an atom and its image by Morawitz ${ }^{15}$ and Milonni and Knight $^{2}$ and assume a two-level system with ground state $|g\rangle$ and excited $|e\rangle$. To deduce the indistinguishability between the atom and its mirror atom, that is, the photon emission 


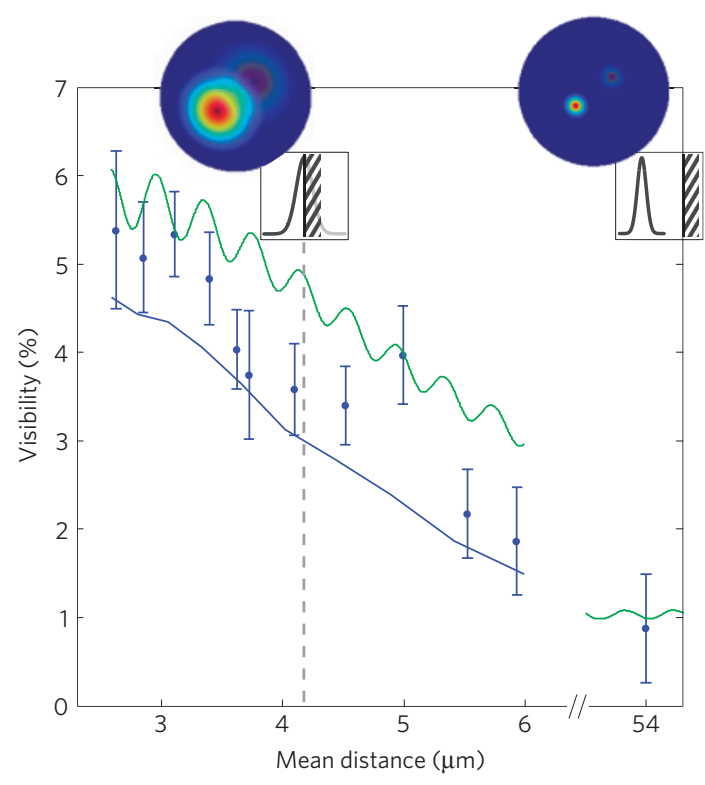

Figure 3 | Dependence of visibility on the mean atom-mirror distance. Measured data is depicted as blue points. The mean distance is calculated from the position of the 'entangling' mirror with respect to the centre of the atomic beam, as indicated in the insets. The error bars indicate a $95 \%$ confidential interval resulting from the fitting procedure to the interference pattern. The expectation from the simple cross-section overlap model is shown by the blue line. The quantum mechanical treatment is depicted as a green line. One finds good agreement between theory and experiment by including details such as initial spatial and momentum distribution,

averaging over all distances, details of Bragg scattering and the final spatial resolution of the atom detector. The mean atom-mirror distance is adjusted by the position of the 'entangling' mirror with respect to the collimation slit of the atomic beam.

towards and away from the mirror, we attribute to the atom a size corresponding the optical absorption cross-section $\left(\sigma=3 \lambda^{2} / 2 \pi\right)$. In the direction perpendicular to the mirror an observer cannot in principle distinguish between the atom and mirror atom and thus a coherent superposition of momentum states $|\psi\rangle=1 / \sqrt{2}$ $\left(\left|+\hbar k_{0}\right\rangle+\left|-\hbar k_{0}\right\rangle\right)$ emerges with the photon momentum $p_{\text {rec }}=\hbar k_{0}$. For emission directions other than perpendicular, the probability $P$ for generating $\left|\psi^{\prime}\right\rangle=1 / \sqrt{2}\left(\left|+\hbar k^{\prime}\right\rangle+\left|-\hbar k^{\prime}\right\rangle\right)$ can be estimated by the overlap region of an atom and mirror atom with the assigned effective size, as shown in Fig. 1b. This overlap depends on the distance between the atom and the mirror and on the observation angle (for details see Supplementary Information). To quantitatively compare with the experimental data, the finite resolution of momentum detection has to be taken into account, leading to an integration over different observation directions. Further averaging due to the finite extension of the atomic beam (width in transverse direction of $10 \mu \mathrm{m}$ ) and the initial momentum distribution results in a reduction of the visibility $V$. The prediction within this model is shown as the solid blue line in Fig. 3.

The comprehensive quantum mechanical model (for details see Supplementary Information) takes into account the modified mode structure of the electromagnetic field due to the presence of the mirror ${ }^{16}$. We derive a master equation for the internal degrees of freedom of the atom and its centre of mass motion perpendicular to the mirror surface. It is found that the quantum state of the atomic centre of mass motion after spontaneous emission can be written as

$$
\hat{\varrho}_{\mathrm{gg}}(t=\infty)=\alpha \frac{3}{8} \int_{0}^{1} \mathrm{~d} u\left(\left|\psi_{\mathrm{s}}\right\rangle\left\langle\psi_{\mathrm{s}}\left|+u^{2}\right| \psi_{\mathrm{p}}\right\rangle\left\langle\psi_{\mathrm{p}}\right|\right)
$$

where

$$
\begin{gathered}
\left|\psi_{\mathrm{s}}\right\rangle=\left(r_{\mathrm{s}}{ }^{*} \mathrm{e}^{i k_{0} u \hat{z}}+\mathrm{e}^{-i k_{0} u \hat{z}}\right)\left|\psi_{0}\right\rangle \\
\left|\psi_{\mathrm{p}}\right\rangle=\left(-r_{\mathrm{p}}{ }^{* i k_{0} u \hat{z}}+\mathrm{e}^{-i k_{0} u \hat{z}}\right)\left|\psi_{0}\right\rangle
\end{gathered}
$$

The operators $e^{ \pm i k_{0} u \hat{z}}$ in equations (1) and (2) describe the transverse recoil momentum $\pm \hbar k_{0} u$ transferred to the atom by the spontaneously emitted photon. The Fresnel coefficient $r_{s}\left(r_{p}\right)$ accounts for the reflection of the transversal electric (transversal magnetic) mode at the mirror and $\left|\psi_{0}\right\rangle$ describes the motional state of the atom before spontaneous emission. The normalization is ensured by the normalization constant $\alpha$. For a quantitative comparison with the experiment we assume that

$$
\left|\psi_{0}\right\rangle=\int \mathrm{d} p f(p, d) \mathrm{e}^{\frac{i}{\hbar} p d} \mathrm{e}^{i \phi_{\mathrm{r}}(p)}|p\rangle
$$

is a coherent wave packet. The quantity $|f(p, d)|^{2}$ represents the initial momentum distribution of atoms and is inferred from an independent measurement of the momentum distribution. The description of the initial atomic state by a pure state is a sensible assumption because the width of the slit collimating the atoms is chosen to be close to the diffraction limit. The phase $\phi_{\mathrm{f}}(p)$ determines the shape of the wavefunction in position space. The Bragg grating is modelled as a beamsplitter with a momentum dependent splitting ratio determined from experimental measurements. After free evolution of the atom we determine the probability to detect the atom within the given resolution of the detector. The result of this calculation is shown as the green line in Fig. 3, where only the phase $\phi_{\mathrm{f}}(p)$ of the wavefunction in front of the first mirror cannot be fully reconstructed acting as a free parameter. The uncertainty of this phase explains the smaller visibility and the asymmetry between different diffraction orders (see Fig. 4).

So far we have discussed the maximum coherence observed in the experiment. In Fig. 4 the momentum dependence of the coherence is shown for a mean distance of $3.3 \mu \mathrm{m}$ from the mirror. This reveals that only the outermost parts of the momentum distribution are in a coherent superposition, which is consistent with the simple picture of an atom and mirror atom. It is important to note that Bragg scattering itself exhibits a momentum dependence (Bragg acceptance). For the chosen short interaction length the Bragg acceptance is indicated by the grey line in Fig. 4 . As the observed coherence decays significantly within the Bragg acceptance we can experimentally confirm that only the most extreme emission events, that is, perpendicular to the mirror surface, lead to a significant generation of coherence. This angular dependence is similar for all investigated mirror distances as it is essentially given by the coherent momentum spread of the strongly confined initial atomic beam.

Finally, we would like to point out the differences from other experiments where the connection between spontaneous emission and coherence has been investigated. For example, the experiment in ref. 8 shows that the spontaneous photon carries away information from the atom about its position, and therefore destroys the coherence when the two paths can be distinguished. Another experiment ${ }^{5}$, on the other hand, provides direct proof for the coherence of photons emitted in the resonance fluorescence of a laser-driven ion in front of a mirror. The observed interference pattern can be regarded as indirect evidence for the motional coherence of the trapped ion, well within the Lamb-Dicke limit ${ }^{6}$. A different example in the context of laser cooling is velocity selective coherent population trapping ${ }^{17}$, where spontaneous emission populates motional dark states. Here the direction of the emitted photon is indistinguishable because it is emitted in the direction of a macroscopic classical field that drives the atom. The most salient 


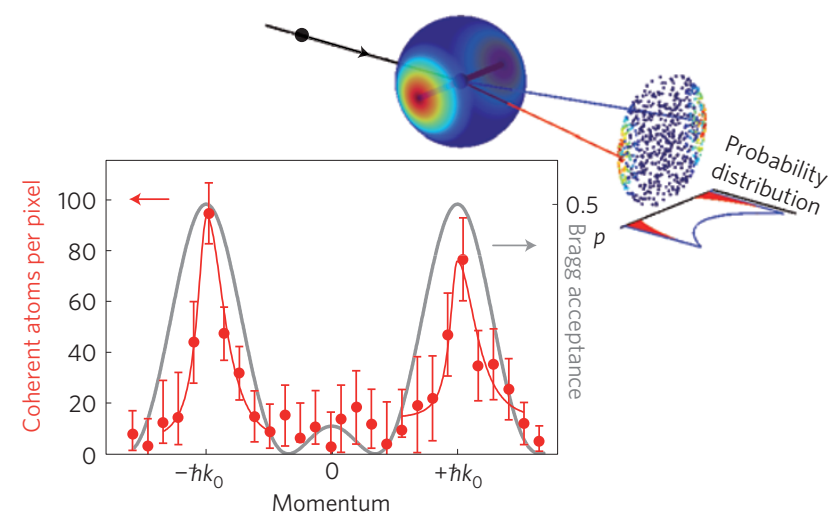

Figure 4 | Observation of angular dependence of coherence. The schematics show an idealized case of coherent momenta for an atom at a fixed distance and an initial momentum parallel to the mirror (red area within the momentum distribution). Owing to the finite momentum distribution of the atomic beam, the narrow coherent momenta is smeared out in the experimental realization. The measured width of the coherent momenta (red points) is smaller than the angle-acceptance of the Bragg-crystal (grey line), revealing that mainly atoms with momenta of $\pm \hbar k_{0}$ are in a coherent superposition. The data is shown for a mean distance of $3.3 \mu \mathrm{m}$ (in contrast to Fig. $2 \mathrm{~b}$ (lower graph), where the atom is much closer to the mirror). Error bars are defined as in Fig. 3.

feature of our experiment is that a single spontaneous emission event in front of a mirror creates a coherent superposition in freely propagating atomic matter-waves, without any external coherent fields being involved. The emission directions of the spontaneous photon become indistinguishable because of the mirror.

In work by Bertet et al. ${ }^{12}$ photons from transitions between internal states are emitted into a high finesse cavity. Their first experiment demonstrates the transition from indistinguishability when emission is into a large classical field to distinguishability and destruction of coherence between the internal atomic states when emission is into the vacuum state of the cavity. In their second experiment they show that, using the same photon for both beamsplitters in an internal state interferometer sequence, coherence can be obtained even in the empty cavity limit. In our experiment the photon leaves the apparatus. We observe coherence only when the photon cannot carry away 'which path' information. This implies that the generated coherence in motional states is robust and lasts. In this sense it is an extension of Einstein's famous recoiling slit Gedanken experiment ${ }^{11}$. The single photon is the ultimate lightweight beamsplitter, which can be in a robust coherent superposition of two motional states. In free space the momentum of the emitted photon allows the measurement of the path of the atom. This corresponds to a well-defined motional state of the beamsplitter (that is, no coherence). Close to the mirror the reflection renders some paths indistinguishable, realizing a coherent superposition of the beamsplitter. The large mass of the mirror ensures that even in principle the photon recoil cannot be seen. Thus, the atom is in a coherent superposition of the two paths. We measure this generated coherence by matter-wave interference.
Received 6 September 2010; accepted 1 March 2011; published online 3 April 2011

\section{References}

1. Milonni, P. W. The Quantum Vacuum (Academic, 1994).

2. Milonni, P. W. \& Knight, P. L. Spontaneous emission between mirrors. Opt. Commun. 9, 119-122 (1973).

3. Drexhage, K. H. Progress in Optics Vol. 12, 163-192, 192a, 193-232 (Elsevier, 1974).

4. Goy, P., Raimond, J., Gross, M. \& Haroche, S. Observation of cavity-enhanced single-atom spontaneous emission. Phys. Rev. Lett. 50, 1903-1906 (1983).

5. Eschner, J., Raab, C., Schmidt-Kaler, F. \& Blatt, R. Light interference from single atoms and their mirror images. Nature 413, 495-498 (2001).

6. Eschner, J. Sub-wavelength resolution of optical fields probed by single trapped ions: Interference, phase modulation, and which-way information. Eur. Phys. J. D 22, 341-345 (2003).

7. Pfau, T., Spälter, S., Kurtsiefer, C., Ekstrom, C. R. \& Mlynek, J. Loss of spatial coherence by a single spontaneous emission. Phys. Rev. Lett. 73, 1223-1226 (1994).

8. Chapman, M. S. et al. Photon scattering from atoms in an atom interferometer: Coherence lost and regained. Phys. Rev. Lett. 75, 3783-3787 (1995).

9. Kokorowski, D. A., Cronin, A. D., Roberts, T. D. \& Pritchard, D. E. From single- to multiple-photon decoherence in an atom interferometer. Phys. Rev. Lett. 86, 2191-2195 (2001).

10. Oberthaler, M. et al. Dynamical diffraction of atomic matter waves by crystals of light. Phys. Rev. A 60, 456-472 (1999).

11. Bohr, N. in Albert Einstein: Philosopher Scientist (ed. Schilpp, P.A.) (Library of Living Philosophers, Evanston, 1949); reprinted in Quantum Theory and Measurement (eds Wheeler, J. A. and Zurek, W. H.) 9-49 (Princeton Univ. Press, 1983).

12. Bertet, P. et al. A complementarity experiment with an interferometer at the quantum-classical boundary. Nature 411, 166-170 (2001).

13. Cronin, A. D., Schmiedmayer, J. \& Pritchard, D. E. Optics and interferometry with atoms and molecules. Rev. Mod. Phys. 81, 1051-1129 (2009).

14. Martin, P., Oldaker, B., Miklich, A. \& Pritchard, D. Bragg scattering of atoms from standing light-wave. Phys. Rev. Lett. 60, 515-518 (1988).

15. Morawitz, H. Self-coupling of a two-level system by a mirror. Phys. Rev. 187, 1792-1796 (1969).

16. Di Stefano, O., Savasta, S. \& Girlanda, R. Three-dimensional electromagnetic field quantization in absorbing and dispersive bounded dielectrics. Phys. Rev. A 61, 023803 (2000).

17. Aspect, A., Arimondo, E., Kaiser, R., Vansteenkiste, N. \& Cohen-Tannoudji, C. Laser cooling below the one-photon recoil energy by velocity-selective coherent population trapping. Phys. Rev. Lett. 61, 826-829 (1988).

\section{Acknowledgements}

We wish to thank Florian Ritterbusch for assistance throughout the preparation of this manuscript. We gratefully acknowledge support from the Forschergruppe FOR760, Deutsche Forschungsgemeinschaft, the German-Israeli Foundation, the Heidelberg Center of Quantum Dynamics, Landesstiftung Baden-Württemberg, the ExtreMe Matter Institute and the European Commission Future and Emerging Technologies Open Scheme project MIDAS (Macroscopic Interference Devices for Atomic and Solid-State Systems). M.K. acknowledges financial support within the framework of the Emmy Noether project HA 5593/1-1 funded by the German Research Foundation (DFG). J.S. acknowledges financial support through the Wittgenstein Prize.

\section{Author contributions}

J.S and M.K.O. conceived the conceptual idea, M.K.O. and J.T. designed the experiment, J.T., M.S. and J.W. performed the experiment, J.T., M.K. and M.K.O. analysed the data, J.T. and M.K.O. developed the semiclassical model, M.K. contributed the quantum mechanical model. All authors discussed the results and wrote the manuscript.

\section{Additional information}

The authors declare no competing financial interests. Supplementary information accompanies this paper on www.nature.com/naturephysics. Reprints and permissions information is available online at http://npg.nature.com/reprintsandpermissions. Correspondence and requests for materials should be addressed to J.T. 